\title{
Polarization of calcium atomic fluorescence due to a coherence effect in the photodissociation of $\mathrm{Ca}_{2}$ molecules $\left(^{*}\right)$
}

\author{
J. Vigué \\ Laboratoire de Spectroscopie Hertzienne de l'ENS $\left({ }^{* *}\right)$, 24, rue Lhomond, 75231 Paris Cedex 05, France \\ P. Grangier, G. Roger and A. Aspect \\ Institut d'Optique (**), Université Paris-Sud, B.P. 43, 91406 Orsay Cedex, France
}

(Rę̧u le 5 octobre 1981, accepté le 30 octobre 1981)

\begin{abstract}
Résumé. - Nous avons observé un taux de polarisation élevé $(64 \%)$ de la lumière de fluorescence émise par des atomes de calcium excités par la photodissociation de molécules de $\mathrm{Ca}_{2}$ dans leur état fondamental. Cette valeur élevée est en contradiction avec les prédictions de Van Brunt et Zare et nous montrons qu'elle est due à des effets de cohérence entre les sous-niveaux de l'état excité.
\end{abstract}

\begin{abstract}
A strong degree of polarization (64\%) has been observed in the fluorescence from calcium atoms excited by the photodissociation of $\mathrm{Ca}_{2}$ molecules in their ground state. This large value, at variance with the predictions by Van Brunt and Zare, is explained by taking into account a coherence effect between the sublevels of the excited state.
\end{abstract}

1. Introduction. - The possibility of polarization of atomic fluorescence excited by molecular dissociation was predicted in 1968 by Van Brunt and Zare [1] (hereafter referred to by VBZ). The first observation of this effect has recently been reported for the photodissociation of $\mathrm{Na}_{2}$ by Rothe et al. [2] who have observed a degree of polarization of minus $5 \%$. In this letter, we report the observation of the $422.7 \mathrm{~nm} 4 \mathrm{~s} 4 \mathrm{p}^{1} \mathrm{P}_{1}-4 \mathrm{~s}^{2}{ }^{1} \mathrm{~S}_{0}$ fluorescent resonance line of atomic calcium, following the photodissociation of $\mathrm{Ca}_{2}$ by the polarized light of the violet krypton laser lines: This atomic fluorescence has been found to be strongly polarized $(64 \%)$. Such a degree of polarization is greater than the maximum value predicted by the VBZ theory : $50 \%$ (or $14 \%$ ) if the $m$ values of the atomic ${ }^{1} \mathrm{P}_{1}$ state selected by the photodissociation process are equal to 0 (or \pm 1 , respectively), the quantization axis being the internuclear axis. We explain our experimental observations by taking into account coherence effects between the sublevels $m= \pm 1$ of the ${ }^{1} P_{1}$ atomic state. The main results of our calculation are the following : the photodissociation is due to an excitation from the ground

(*) La version française de cet article a été proposée pour publication aux Comptes Rendus de l'Académie des Sciences.

$\left({ }^{* *}\right)$ Associé au C.N.R.S
$\mathrm{X}^{1} \Sigma_{\mathrm{g}}^{+}$to a ${ }^{1} \Pi_{\mathrm{u}}$ state, which yields by dissociation one ${ }^{1} S_{0}$ atom and one ${ }^{1} P_{1}$ atom in the states \pm 1 , these two states being occupied coherently; then the maximum degree of polarization is as high as $78 \%$ and the degree of polarization remains quite large $(60 \%)$ if the spatial distribution of the dissociation products is isotropic.

2. Experimental. - Figure 1 shows a plan of the experimental set-up partially described elsewhere [3]. A molecular beam of calcium is irradiated by the $406 \mathrm{~nm}$ or $413 \mathrm{~nm}$ violet line provided by a linearly polarized C.W. krypton ion laser, focused at the interaction region (beam waist radius at $1 / e^{2} \simeq 30 \mu \mathrm{m}$ ). The fluorescent light is collected by a wide aperture aspherical lens $(f / 0.8)$ with its axis in a direction perpendicular to the laser polarization. It is then filtered either by a monochromator or by a narrow band interference filter ( 2 nm f.w.h.m.) centred at $422.7 \mathrm{~nm}$. The detection is carried out by standard photon counting techniques : a typical signal value is 1000 counts/s while the background (with the molecular beam cut off by a flag) is $300 \mathrm{c} / \mathrm{s}$. The overall detection efficiency was determined by a technique using a radiative cascade and two photon coincidence measurements [3]. It was found to be about $3 \times 10^{-3}$ with the interference filter in place. 


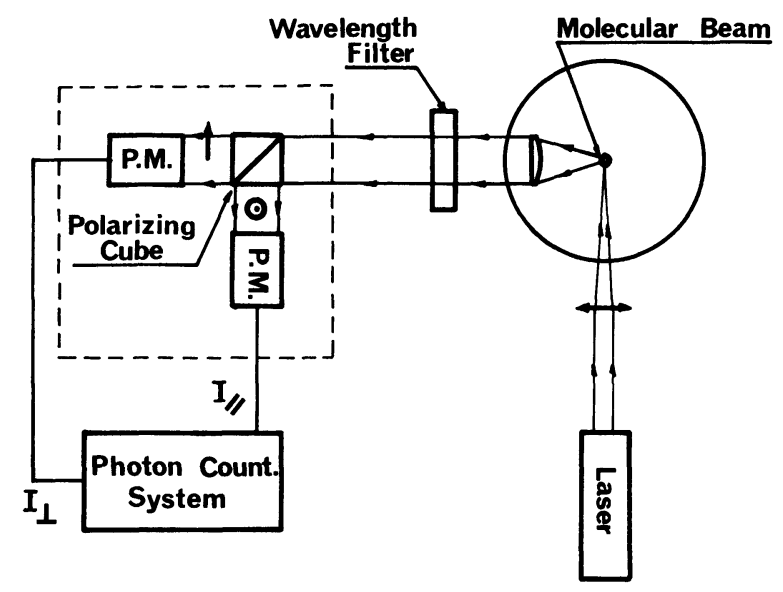

Fig. 1. - The molecular beam and the laser polarization are both perpendicular to the figure plane. The polarimeter device (polarizing cube and photomultipliers) can rotate around the axis of the detection channel.

We can insert a polarizing cube separating the light component, parallel and perpendicular to the laser polarization, and measure the intensities $I_{\|}$ and $I_{\perp}$. We thus obtain a direct measurement of the degree of polarization $P=\left(I_{\|}-I_{\perp}\right) /\left(I_{\|}+I_{\perp}\right)$. The equality of the detection efficiencies for $I_{\|}$and $I_{\perp}$ is checked by a $90^{\circ}$ rotation of the polarimeter device (cube and photomultipliers) around the axis of the detection channel.

The molecular beam is produced by heating calcium in an oven between $900 \mathrm{~K}$ and $1050 \mathrm{~K}$. Since there are no collisions in the beam, all the molecules are produced in the oven. Their density is related to the atomic density by the law

$$
\left[N_{\mathrm{Ca}_{2}}\right] \cdot\left[N_{\mathrm{Ca}}\right]^{-2}=K(T) .
$$

We have calculated $K(T)$ by a standard statistical mechanics formula [4] using the ground state $\mathrm{X}^{1} \Sigma_{\mathrm{g}}^{+}$ constants. $\left[N_{\mathrm{Ca}}\right]$ is easily related to the tabulated data for calcium vapour pressure (see reference [3]). The molecular density at the interaction region (at $10 \mathrm{~cm}$ from the oven exit) is then derived by gas kinetics calculations. At $1000 \mathrm{~K}$, we calculate a density of $1.9 \times 10^{9}$ molecules $/ \mathrm{cm}^{3}$ in the oven and $3.2 \times 10^{5}$ molecules $/ \mathrm{cm}^{3}$ in the interaction region.

3. Results and discussion. - The $422.7 \mathrm{~nm}$ $4 s 4 p{ }^{1} P_{1}-4 s^{2}{ }^{1} S_{0}$ resonance line of atomic calcium has been observed with laser excitation at both wavelengths 406 and $413 \mathrm{~nm}$. As shown in figure 2, the signal is proportional to the molecular density at a laser power of 0.2 watt. At this power, the dissociated fraction of molecules is estimated to be roughly $4 \%$ with $406 \mathrm{~nm}$ and $2 \%$ with $413 \mathrm{~nm}$. We interpret these observations as evidence for a photodissociation of the $\mathrm{Ca}_{2}$ molecules, yielding an excited $4 \mathrm{~s} 4 \mathrm{p}^{1} \mathrm{P}_{1}$ atom and another atom in its ${ }^{1} S_{0}$ ground state [6]. In the interaction region $\left(10^{-4} \mathrm{~s}\right.$ after effusing from the oven) all the molecules are in the truly bound rovi-

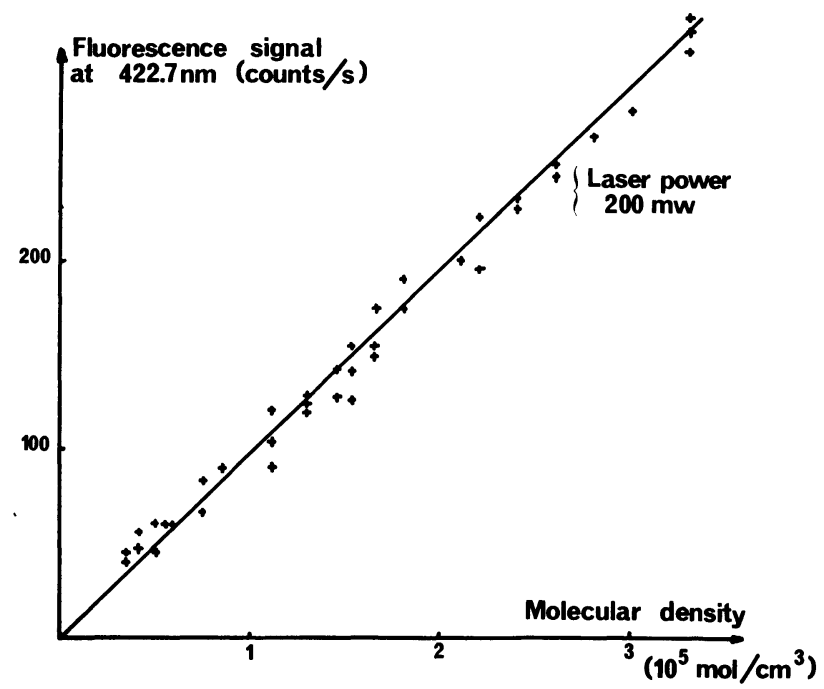

Fig. 2. - Atomic fluorescence signal $(422.7 \mathrm{~nm}$ line) $v s$. molecular density at the interaction region.

brational levels of the $\mathrm{X}^{1} \Sigma_{\mathrm{g}}^{+}$ground state. The thermodynamical threshold for dissociation is given by

$$
E(v, J)>D_{\mathrm{e}}^{\prime \prime}+E\left({ }^{1} \mathrm{P}_{1}\right)-h v
$$

where $E(v, J)$ is the energy of the initial state, $D_{\mathrm{e}}^{\prime \prime}=1095 \mathrm{~cm}^{-1}$ is the dissociation energy of the molecular ground state [5], and $E\left({ }^{1} \mathrm{P}_{1}\right)=23652 \mathrm{~cm}^{-1}$ is the atomic energy of the excited atom. This leads to $E(v, J)>549 \mathrm{~cm}^{-1}$ for an excitation at $413 \mathrm{~nm}$, and $E(v, J)>168 \mathrm{~cm}^{-1}$ for an excitation at $406 \mathrm{~nm}$. With $K T$ about $700 \mathrm{~cm}^{-1}$, a large population fulfills these conditions. On the other hand there is not enough energy available for yielding two excited atoms. The molecular states correlating with the $4 s 4 p^{1} P_{1}+4 s^{2}{ }^{1} S_{0}$ dissociation products are ${ }^{1} \Sigma_{g}^{+}$, ${ }^{1} \Pi_{\mathrm{g}},{ }^{1} \Sigma_{\mathrm{u}}^{+}$and ${ }^{1} \Pi_{\mathrm{u}}[7]$. Among them, only ${ }^{1} \Sigma_{\mathrm{u}}^{+}$and ${ }^{1} \Pi_{\mathrm{u}}$ can be excited from the $\mathrm{X}^{1} \Sigma_{\mathrm{g}}^{+}$ground state. The ${ }^{1} \Pi_{\mathrm{u}} \leftarrow{ }^{1} \Sigma_{\mathrm{g}}^{+}$system has been recently observed in $\mathrm{Ca}_{2}$ isolated in a rare gas matrix [8].

The degree of polarization of the atomic fluorescence has been found to be independent of the laser power in a range between $25 \mathrm{~mW}$ and $300 \mathrm{~mW}$, and also independent of the molecular density. For a $406 \mathrm{~nm}$ excitation, the average of 12 measurements of $10 \mathrm{~s}$ counting time gives :

$$
P(406 \mathrm{~nm})=0.64 \pm 0.01 \text {. }
$$

The dispersion of the results is in agreement with a Poisson law for photon counting and we have taken as an error the corresponding standard deviation of the average. For an excitation at $413 \mathrm{~nm}$ the degree of polarization was found to be slightly larger (about 0.68 ) but the precision of the result is not so good since some stray light at $413 \mathrm{~nm}$ due to Rayleigh scattering of the laser beam by the calcium atoms was not completely eliminated by the interference filter. 
Depending on whether the excited dissociating state is ${ }^{1} \Sigma_{\mathrm{u}}^{+}$or ${ }^{1} \Pi_{\mathrm{u}}$, the atomic $m_{l}$ selected by the dissociation process are $m_{l}=0$ or $m_{l}= \pm 1$, as shown by VBZ. According to their calculation, the degree of polarization cannot exceed $50 \%$ in the first case and $14 \%$ in the second. Our measurements clearly contradict these predictions. But a calculation taking into account coherence effects between the $m_{l}= \pm 1$ sublevels (see $\S 4$ ) predicts a maximum degree of polarization of $78 \%$. As the calculation of VBZ remains valid if the selected $m_{l}$ value is $m_{l}=0$, we are thus led to the conclusion that the dissociating state of $\mathrm{Ca}_{2}$ produces $m_{l}= \pm 1$ sublevels and is therefore a ${ }^{1} \Pi_{\mathrm{u}}$ state. The difference between the measured degree of polarization $(64 \%)$ and the maximum predicted by our calculation $(78 \%)$ is attributed to several factors. The large aperture of the collecting lens accounts for a $3 \%$ reduction. Some molecular rotation can reduce the spatial anisotropy of the distribution of the dissociation products and at the same time $P$ (see Fig. 4). Another possible reduction can be due to a saturation of the excitation process [9]. However since the dissociating ${ }^{1} \Pi_{\mathrm{u}}$ state is almost completely unknown, and as many rovibrational levels of the ground state are involved in the photodissociation we cannot give a quantitative treatment of the two last effects. This treatment could also give an explanation of the different degrees of polarization for the two exciting wavelengths 406 and $413 \mathrm{~nm}$.

4. Calculation of the polarization of the atomic fluorescence, taking into account coherence effects. The first step of the calculation consists in finding the molecular state reached after the excitation, and in evaluating the probability of this process, as a function of the relative orientations of the molecular axis and the laser polarization. We only consider the cases where $\Lambda$ (projection of the orbital electronic momentum on the molecular axis) is different from 0 . When $\Lambda=0$, no coherence effects can occur, and the VBZ calculation holds in all the cases.

In order to simplify the calculation, we neglect the molecular rotation. We thus use, as molecular wave function basis, the set $|\Lambda, \theta, \varphi\rangle$ where $\theta$ and $\varphi$ specify the direction of the molecular axis (Fig. 3). We omit the spins since all the levels involved are singlet. With a proper choice of the phases of this set, the states in a given $\sigma_{\mathrm{v}}$ parity (reflection by the plane $(x O z))$ are

$\left|\tilde{\Lambda}^{ \pm}, \theta, \varphi\right\rangle=(1 / \sqrt{2})\left[|\Lambda, \theta, \varphi\rangle^{ \pm}|-\Lambda, \theta, \varphi\rangle\right]$.

The components of the molecular electric dipole D in the molecular frame are even $\left(D_{x}\right.$ and $\left.D_{z}\right)$ or odd $\left(D_{y}\right)$ with respect to $\sigma_{\mathrm{v}}$. The electric field of the excitation laser being along $O Z$, the probability of an

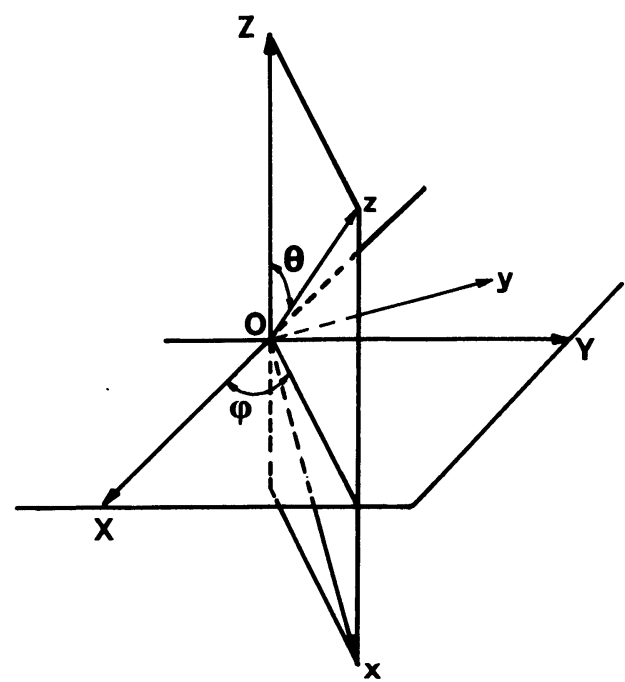

Fig. 3. - The molecular axis $O z$ (unit vector $\mathbf{k}$ ) is characterized by the angles $\theta$ and $\varphi$ with respect to the laboratory frame $O X Y Z$. The laser beam is polarized along $O Z$. The axis $O x$ (unit vector i) is perpendicular to $O z$ and in the plane $(O z, O Z$ ), while $O y$ (unit vector $\mathbf{j}$ ) is the third axis of the molecular frame.

excitation from the $\mathrm{X}$ ground state to a $\tilde{\Lambda}^{ \pm}$state involves the matrix elements

$$
\left\langle\mathrm{X}, \theta, \varphi\left|D_{z}\right| \tilde{\Lambda}^{ \pm}, \theta, \varphi\right\rangle
$$

since

$$
D_{z}=-D_{x} \sin \theta+D_{z} \cos \theta .
$$

$D_{Z}$ is even with respect to $\sigma_{\mathrm{v}}$. In the case of $\mathrm{Ca}_{2}$, the ground state is a + state and only a $\left|\Lambda^{+}, \theta, \varphi\right\rangle$ state can be excited. For such a state the wave function for a large internuclear distance $r$ between the atoms $a$ and $b$ is [10]

$$
\begin{aligned}
\left|\tilde{\Lambda}^{+}, \theta, \varphi\right\rangle \underset{r \rightarrow \infty}{\sim} \frac{1}{v 2}\left(\left|\tilde{l}^{+}\right\rangle_{\mathrm{a}}\left|{ }^{1} \mathrm{~S}_{0}\right\rangle_{\mathrm{b}}+\right. \\
\left.\left.\quad+\left.\varepsilon\right|^{1} \mathrm{~S}_{0}\right\rangle_{\mathrm{a}}\left|\tilde{l}^{+}\right\rangle_{\mathrm{b}}\right)
\end{aligned}
$$

where $|\varepsilon|=1$. Equation (4) takes into account the fact that one of the two atoms is left in a ground state with a null angular momentum and we assume that there is no mixing between Born Oppenheimer states. The excited atom is therefore left in a pure state :

$$
\left|\tilde{l}^{+}\right\rangle=\frac{1}{\sqrt{2}}\left(|l, \Lambda\rangle+(-1)^{\Lambda}|l,-\Lambda\rangle\right)
$$

(the quantization axis is the internuclear axis $\mathrm{Oz}$ and the $(-1)^{A}$ term comes from the symmetry of spherical harmonics with respect to $\sigma_{\mathrm{v}}$ ).

The preceding calculation also yields the spatial dependence $f(\theta, \varphi)$ of the excitation probability, as a function of the orientation of the molecular axis. By use of equation (3), one obtains

$$
f(\theta, \varphi)=\sin ^{2} \theta \quad \text { if } \quad \Lambda=1
$$


which is indeed a particular case $(\beta=-1)$ of the general form considered by VBZ

$$
f(\theta)=1+\beta \cdot P_{2}(\cos \theta)
$$

with

$$
P_{2}(\cos \theta)=(1 / 2) \cdot\left(3 \cos ^{2} \theta-1\right) .
$$

Assuming that the initial distribution of molecular axes is isotropic, $f(\theta, \varphi)$ represents also the spatial distribution of molecular axes of the dissociated molecules.

Let us now calculate the polarization of the fluorescence light emitted by an atom in a transition from the state $\left|\tilde{l}^{+}\right\rangle$defined by equation (5) to a state of orbital angular momentum $l^{\prime}$. The coherence between $m_{l}$ and $-m_{l}$ sublevels will be detected on this polarization only if $\left|m_{l}\right|=\Lambda=1$ as it is well known that polarization measurements are not sensitive to coherences with $\Delta m>2$. The fluorescence intensity detected with a polarization $\mathbf{e}_{2}$ in a transition from $\left|\tilde{l}^{+}\right\rangle$to all the sublevels $m^{\prime}$ of $l^{\prime}$ is proportional to

$$
I\left(\mathbf{e}_{\lambda}\right)=\sum_{m^{\prime}}\left|\left\langle l^{\prime}, m^{\prime}\left|\mathbf{e}_{\lambda} \cdot \mathbf{D}\right| \tilde{l}^{+}\right\rangle\right|^{2}
$$

where $\mathbf{D}$ is the atomic electric dipole. By use of Racah formalism one obtains :

$$
\begin{gathered}
I\left(\mathbf{e}_{\lambda}\right)=\left|\left\langle l^{\prime}\|D\| l\right\rangle\right|^{2} \\
\sum_{m^{\prime}} \mid \sum_{q}(-1)^{l^{\prime}-m^{\prime}+q} \times \\
\times\left. C_{\lambda-q}\left[\left(\begin{array}{ccc}
l^{\prime} & 1 & l \\
-m^{\prime} & q & \Lambda
\end{array}\right)+(-1)^{\Lambda}\left(\begin{array}{ccc}
l^{\prime} & 1 & l \\
-m^{\prime} & q & -\Lambda
\end{array}\right)\right]\right|^{2}
\end{gathered}
$$

where $\left\langle l^{\prime}\|D\| l\right\rangle$ is the reduced matrix element for this transition and the $C_{\lambda q}$ are the standard components of $e_{\lambda}$ in the molecular frame

$$
C_{\lambda 0}=\mathbf{k} . \mathbf{e}_{\lambda} \quad C_{\lambda \pm 1}=\mp(2)^{-1 / 2} \mathbf{e}_{\lambda} \cdot(\mathbf{i} \pm i \mathbf{j})
$$

(i, $\mathbf{j}, \mathbf{k}$ being the unit vectors on $O x, O y, O z$ ).

If we particularize to the present case $\left({ }^{1} \mathrm{P} \rightarrow{ }^{1} \mathrm{~S}\right.$ transition $l=\Lambda=1, l^{\prime}=m^{\prime}=0$ ) equation (9) reduces to

$$
I\left(\mathbf{e}_{\lambda}\right)=I_{0}\left|C_{\lambda 1}-C_{\lambda-1}\right|^{2}
$$

exhibiting clearly the interference effect between $C_{\lambda 1}$ and $C_{\lambda-1}$. The polarization of the light emitted in the photodissociation will then be calculated by averaging over all the possible orientations of the internuclear axis $\mathrm{Oz}$ when the fluorescence occurs. Considering that the molecular rotation is negligible during the photodissociation process, the spatial distribution of $\mathrm{Oz}$ is given by equation (6) : $f(\theta, \varphi)=\sin ^{2} \theta$. For an emission in a direction perpendicular to the laser polarization $O Z$, the intensities of the components parallel and perpendicular to $O Z$ are

$$
\begin{aligned}
& I_{\|}=I_{0} \int f(\theta, \varphi) \sin ^{2} \theta \mathrm{d} \Omega \\
& I_{\perp}=I_{0} \int f(\theta, \varphi) \cos ^{2} \theta \cos ^{2} \varphi \mathrm{d} \Omega
\end{aligned}
$$

with $\mathrm{d} \Omega=\sin \theta \mathrm{d} \theta \mathrm{d} \varphi$. The degree of polarization $P$ is given by :

$$
P=\frac{I_{\|}-I_{\perp}}{I_{\|}+I_{\perp}}=\frac{7}{9} \simeq 0.78 .
$$

It seems obvious that if some molecular rotation occurs, its effect will be to reduce the degree of polarization $P$. It is not possible to evaluate in a consistent manner the effect of rotation at this point of our calculation. We may however get a rough idea of its effect by using the method of VBZ; we replace in equation (11) $f(\theta, \varphi)$ by its general form given by equation (7). The degree of polarization $P$ thus calculated is simply :

$$
P=\frac{I_{\|}-I_{\perp}}{I_{\|}+I_{\perp}}=\frac{15-6 \beta}{25-2 \beta} .
$$

Figure 4 depicts the dependence of $P$ as a function of $\beta$; it appears clearly that the coherence effect has increased dramatically the degree of polarization. Particularly, even if the spatial distribution of dissociation products is completely isotropic $(\beta=0)$ the atomic fluorescence remains strongly polarized $(P=60 \%)$.

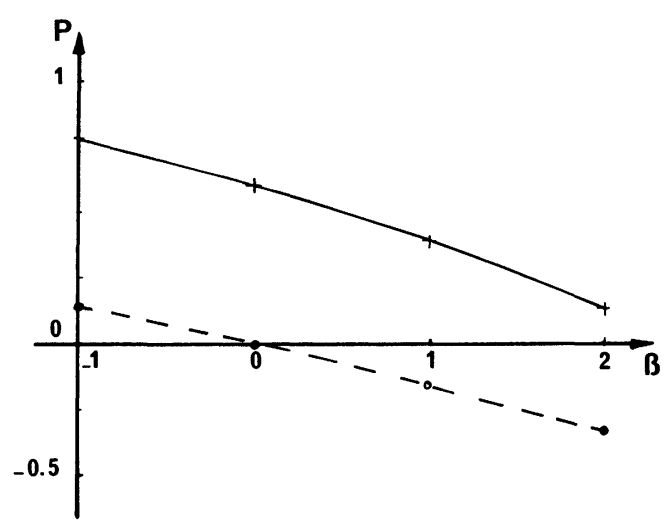

Fig. 4. - Degree of polarization $P$ as a function of the asymmetry parameter $\beta$ for a ${ }^{1} \mathrm{P}_{1}, m_{l}= \pm 1 \rightarrow{ }^{1} \mathrm{~S}_{0}$ transition. The solid curve takes into account the coherence effect between $m_{l}= \pm 1$ sublevels, while the dotted curve neglects such effects. In the presence of a coherence effect, $P$ does not vanish even when $\beta=0$ (no asymmetry).

However, we feel that all the effects of rotation are not taken into account in this calculation. In particular, molecular rotation may introduce a loss of coherence which will decrease the degree of polarization more rapidly than expressed by equation (12).

As a final comment, let us remark that the same result is obtained by a semi-classical calculation considering non-rotating molecules with a polarizabi- 
lity perpendicular to their axis, yielding an atom carrying an oscillating dipole parallel to the polarization of the molecule $(O x)$. Such an oscillating dipole along $O x$, equivalent to two counter rotating coherent dipoles around $\mathrm{O} z$, gives a clear picture of the pure $\left|\tilde{l}^{+}\right\rangle$state involving a linear superposition of the two states $\left|l=1, m_{l}=+1\right\rangle$ and $\mid l=1$, $\left.m_{l}=-1\right\rangle$.

5. Conclusion. - This paper gives the first experimental evidence of a strong polarization of atomic fluorescence following photodissociation. The interpretation of this experimental data has been possible only by taking into account the large coherence between the atomic sublevels populated by photodissociation

Acknowledgments. - We want to thank J. Berlande, M. Broyer, J. C. Lehmann, S. Goursaud and M. Tadjeddine for helpful discussions and advices. We are grateful to J. Durup and J. A. Beswick for interesting comments on the manuscript.

\section{References and footnotes}

[1] Van Brunt, R. J. and Zare, R. N., J. Chem. Phys. 48 (1968) 4304.

[2] Rothe, E. W., Krause, U. and Düren, R., Chem. Phys. Lett. 72 (1980) 100

[3] AsPect, A., Imbert, C. and Roger, G., Opt. Commun. 34 (1980) 46.

[4] Pecker, J. C. and Schatzman, E., Astrophysique générale (Masson, Paris) 1959, p. 59

[5] Vidal, C. R., J. Chem. Phys. 72 (1980) 1864.

We have used the data of this paper, although there is a discrepancy with other work : Wyss, J. C., J. Chem. Phys. 71 (1979) 2949.

[6] Some atomic processes, candidates for yielding fluorescence at the atomic wavelength following excitation at the laser wavelength, have been considered and eliminated. resonance fluorescence triplet or Raman processes would have been proportional to the atomic density instead of the molecular density; laser induced collisions is several orders of magnitude weaker, and would have been weaker at $406 \mathrm{~nm}$ excitation than at $413 \mathrm{~nm}$ excitation.

[7] Stevens, W. J. and Krauss, M., J. Chem. Phys. 67 (1977) 1977.

[8] Miller, J. C., Mowery, R. L., Krausz, E. R., Kim, H. W., Schatz, P. N. and Andrews, L., J. Chem. Phys. 74 (1981) 6349

[9] The fluorescent signal exhibits some saturation for a laser power over $200 \mathrm{~mW}$, showing that a fraction of the transitions are saturated.

[10] Umanskij, S. Ja. and Nikitin, E. E., Theoret. Chim. Acta. $13(1969) 91$ 